\title{
Fuzzy Stability of the Generalized Version of Drygas Functional Equation
}

\author{
Chang Il Kim, ${ }^{1}$ Giljun Han, ${ }^{1}$ and Seong-A Shim ${ }^{2}$ \\ ${ }^{1}$ Department of Mathematics Education, Dankook University, 152 Jukjeon-ro, Suji-gu, Yongin-si, Gyeonggi-do 448-701, \\ Republic of Korea \\ ${ }^{2}$ Department of Mathematics, Sungshin Women's University, 249-1 Dongseon-Dong 3-Ga, Seongbuk-gu, Seoul 136-742, \\ Republic of Korea
}

Correspondence should be addressed to Giljun Han; gilhan@dankook.ac.kr

Received 4 February 2014; Accepted 5 April 2014; Published 27 April 2014

Academic Editor: Roberto Natalini

Copyright (C) 2014 Chang Il Kim et al. This is an open access article distributed under the Creative Commons Attribution License, which permits unrestricted use, distribution, and reproduction in any medium, provided the original work is properly cited.

We consider the functional equation $f(a x+y)+f(a x-y)-a(a+1) f(x)-a(a-1) f(-x)-[f(y)+f(-y)]+k[f(x+y)+f(x-$ $y)-2 f(x)-f(y)-f(-y)]=0$ for a fixed rational number $a$ with $a \neq 1,-1,0$ and a fixed real number $k$. We study the solution of the equation between linear spaces and prove the generalized Hyers-Ulam stability for it when the target space is a fuzzy normed space.

\section{Introduction and Preliminaries}

In 1940, Ulam proposed the following stability problem (cf. [1]):

"Let $G_{1}$ be a group and $G_{2}$ a metric group with the metric $d$. Given a constant $\delta>0$, does there exist a constant $c>0$ such that if a mapping $f$ : $G_{1} \rightarrow G_{2}$ satisfies $d(f(x y), f(x) f(y))<c$ for all $x, y \in G_{1}$, then there exists a unique homomorphism $h: G_{1} \rightarrow G_{2}$ with $d(f(x), h(x))<\delta$ for all $x \in G_{1}$ ?"

In the next year, Hyers [2] gave a partial solution of Ulam's problem for the case of approximate additive mappings. Subsequently, his result was generalized by Aoki [3] and Moslehian and Rassias [4] for additive mappings, and by Rassias [5] for linear mappings, to consider the stability problem with unbounded Cauchy differences. During the last decades, the stability problems of functional equations have been extensively investigated by a number of mathematicians (see [6-10]).

Recently, the stability problems in the fuzzy spaces have been extensively studied (see [11-13]). The concept of fuzzy norm on a linear space was introduced by Katsaras [14] in 1984. Later, Cheng and Mordeson [15] gave a new definition of a fuzzy norm in such a manner that the corresponding fuzzy metric is of Kramosil and Michálek type [16]. In 2008, for the first time, Mirmostafaee and Moslehian [12, 13] used the definition of a fuzzy norm in [17] to obtain a fuzzy version of stability for the Cauchy functional equation

$$
f(x+y)=f(x)+f(y),
$$

and the quadratic functional equation

$$
f(x+y)+f(x-y)=2 f(x)+2 f(y) .
$$

We call a solution of (1) an additive mapping and a solution of (2) is called a quadratic mapping. Also, the equation

$$
\begin{aligned}
T_{f}(x, y)= & f(x+y)+f(x-y) \\
& -2 f(x)-f(y)-f(-y)=0
\end{aligned}
$$

is called Drygas functional equation (see $[18,19]$ for details).

Najati and Moghimi [20] investigated the generalized Hyers-Ulam stability for functional equation derived from additive and quadratic functions on quasi-Banach spaces. 
In this paper, we introduce the following functional equation for a fixed rational number $a$ with $a \neq 1,-1,0$ and a fixed real number $k$ :

$$
\begin{gathered}
f(a x+y)+f(a x-y)-a(a+1) f(x) \\
-a(a-1) f(-x)-[f(y)+f(-y)] \\
+k[f(x+y)+f(x-y) \\
-2 f(x)-f(y)-f(-y)]=0
\end{gathered}
$$

with $f(0)=0$. It is easy to see that the function $f(x)=$ $p x^{2}+q x$ is a solution of the functional equation (4), so we can expect that a solution of (4) is additive-quadratic type. We note that the left-hand side of (4) is essentially the sum of two Drygas functionals $T_{f}(a x, y)$ and $k T_{f}(x, y)$. In Section 2, a complete characterization of the solution of (4) is given. In Section 3, we prove the stability for (4) in fuzzy Banach spaces. One can find some kinds of gaps for finding $r$ in Theorems 13 and 14. In Theorem 15, we resolve these gaps for special and practical case of $\phi(x, y)$. Also, we give an example related to Theorem 15 . We list some definitions related to fuzzy normed spaces.

Definition 1. Let $X$ be a real vector space. A function $N: X \times$ $\mathbb{R} \rightarrow[0,1]$ is called a fuzzy norm on $X$ if for all $x, y \in X$ and all $s, t \in \mathbb{R}$,

(N1) $N(x, t)=0$ for $t \leq 0$;

(N2) $x=0$ if and only if $N(x, t)=1$ for all $t>0$;

(N3) $N=(c x, t)=N(x, t /|c|)$ if $c \neq 0$;

(N4) $N=(x+y, s+t) \geq \min \{N(x, s), N(y, t)\}$;

(N5) $N(x, \cdot)$ is a nondecreasing function of $\mathbb{R}$ and $\lim _{t \rightarrow \infty} N(x, t)=1$;

(N6) for any $x \neq 0, N(x, \cdot)$ is continuous on $\mathbb{R}$.

In this case, the pair $(X, N)$ is called a fuzzy normed space.

Definition 2. Let $(X, N)$ be a fuzzy normed space. A sequence $\left\{x_{n}\right\}$ in $X$ is said to be convergent if there exists an $x \in X$ such that $\lim _{n \rightarrow \infty} N\left(x_{n}-x, t\right)=1$ for all $t>0$. In this case, $x$ is called the limit of the sequence $\left\{x_{n}\right\}$ in $X$ and one denotes it by $N-\lim _{n \rightarrow \infty} x_{n}=x$.

Definition 3. Let $(X, N)$ be a fuzzy normed space. A sequence $\left\{x_{n}\right\}$ in $X$ is said to be Cauchy if for any $\epsilon>0, t>0$, there is an $m \in N$ such that for any $n \geq m$ and any positive integer $p$, $N\left(x_{n+p}-x_{n}, t\right)>1-\epsilon$.

It is well known that every convergent sequence in a fuzzy normed space is Cauchy. A fuzzy normed space is said to be complete if each Cauchy sequence in it is convergent and the complete fuzzy normed space is called a fuzzy Banach space.

\section{Solution of (4)}

In this section, we investigate solutions of (4) between linear spaces $X$ and $Y$ by separating cases into odd functions and even functions. And then, in Theorem 8, it can be concluded that any solution of (4) is additive-quadratic type. We start with the odd function case.

Lemma 4. Let $f: X \rightarrow Y$ be an odd mapping with $f(0)=$ 0 satisfying (4). Suppose that $k \neq-a$. Then $f$ is an additive mapping.

Proof. Since $f$ is an odd mapping, the functional equation (4) can be written by

$$
\begin{aligned}
& f(a x+y)+f(a x-y)-2 a f(x) \\
& \quad+k[f(x+y)+f(x-y)-2 f(x)]=0,
\end{aligned}
$$

for all $x, y \in X$.

If $k=0$, then it is easy to check that $f$ is additive. Suppose that $k \neq 0$. Letting $y=0$ in (5), we have

$$
f(a x)=a f(x),
$$

for all $x \in X$. Replacing $y$ by $x+y$ in (5), we have

$$
\begin{aligned}
& f((a+1) x+y)+f((a-1) x-y) \\
& \quad-2 a f(x)+k[f(2 x+y)-f(y)-2 f(x)]=0,
\end{aligned}
$$

for all $x, y \in X$; letting $y=-y$ in (7), we have

$$
\begin{gathered}
f((a+1) x-y)+f((a-1) x+y)-2 a f(x) \\
+k[f(2 x-y)+f(y)-2 f(x)]=0,
\end{gathered}
$$

for all $x, y \in X$. Replacing $x$ and $y$ by $x+(1 / a) y$ and $x$ in (5), respectively, by (6), we have

$$
\begin{gathered}
a[f((a+1) x+y)+f((a-1) x+y)]-2 a f(a x+y) \\
+k[f(2 a x+y)+f(y)-2 f(a x+y)]=0,
\end{gathered}
$$

for all $x, y \in X$. Letting $y=-y$ in (9), we have

$$
\begin{gathered}
a[f((a+1) x-y)+f((a-1) x-y)]-2 a f(a x-y) \\
+k[f(2 a x-y)-f(y)-2 f(a x-y)]=0,
\end{gathered}
$$

for all $x, y \in X$, and letting $x=2 x$ in (5), we have

$$
\begin{aligned}
& f(2 a x+y)+f(2 a x-y)-2 a f(2 x) \\
& \quad+k[f(2 x+y)+f(2 x-y)-2 f(2 x)]=0,
\end{aligned}
$$

for all $x, y \in X$. By (5), (7), (8), (9), (10), and (11), we have

$$
\begin{aligned}
k(a+k)[f(2 x+y)+f(2 x-y)-2 f(2 x)] \\
-2 k(a+k)[f(x+y)+f(x-y)-2 f(x)]=0,
\end{aligned}
$$

for all $x, y \in X$. If $k \neq-a$, then by (12), we have

$$
\begin{aligned}
& f(2 x+y)+f(2 x-y)-2 f(2 x) \\
& \quad-2 f(x+y)-2 f(x-y)+4 f(x)=0,
\end{aligned}
$$

for all $x, y \in X$ and $f$ is additive-cubic ([20]). Since $f(a x)=$ af $(x)$ for all $x \in X, f$ is additive. 
Lemma 5. Let $f: X \rightarrow Y$ be an odd mapping with $f(0)=$ 0 satisfying (4). Suppose that $k=-a$. Then $f$ is an additive mapping.

Proof. Since $f$ is an odd mapping, the functional equation (4) can be written by

$$
f(a x+y)+f(a x-y)-a[f(x+y)+f(x-y)]=0,
$$

for all $x, y \in X$. Replacing $y$ by $x+y$ in (14), we have

$$
\begin{gathered}
f((a+1) x+y)+f((a-1) x-y) \\
-a[f(2 x+y)-f(y)]=0,
\end{gathered}
$$

for all $x, y \in X$, and interchanging $x$ and $y$ in (15), we have

$$
\begin{gathered}
f(x+(a+1) y)-f(x-(a-1) y) \\
-a[f(x+2 y)-f(x)]=0,
\end{gathered}
$$

for all $x, y \in X$. Replacing $x$ and $y$ by $x+y$ and ay in (5), respectively, we have

$$
\begin{gathered}
f(x+(a+1) y)+f(x-(a-1) y) \\
-f(x+2 y)-f(x)=0,
\end{gathered}
$$

for all $x, y \in X$. By (16) and (17), we have

$$
2 f(x+(a+1) y)-(a+1) f(x+2 y)+(a-1) f(x)=0,
$$

for all $x, y \in X$.

Replacing $x$ and $y$ by $y / a$ and $a x+y$ in (5), respectively, we have

$$
\begin{aligned}
& f(a x+2 y)-f(a x) \\
& \quad-a\left[f\left(a x+\frac{a+1}{a} y\right)-f\left(a x+\frac{a-1}{a} y\right)\right]=0,
\end{aligned}
$$

for all $x, y \in X$. Letting $y=a y$ in (19), we have

$$
\begin{aligned}
f(x+2 y)-f(x) & \\
& -[f(a x+(a+1) y)-f(a x+(a+1) y)]=0,
\end{aligned}
$$

for all $x, y \in X$. Replacing $x$ by $x+y$ in (14), we have

$$
\begin{gathered}
f(a x+(a+1) y)+f(a x+(a-1) y) \\
-a[f(x+2 y)+f(x)]=0,
\end{gathered}
$$

for all $x, y \in X$ and by (20) and (21), we have

$$
2 f(a x+(a+1) y)-(a+1) f(x+2 y)+(a-1) f(x)=0,
$$

for all $x, y \in X$. Replacing $x$ by ax in (18), we have

$$
\begin{gathered}
2 f(a x+(a+1) y)-(a+1) f(a x+2 y) \\
+a(a-1) f(x)=0,
\end{gathered}
$$

for all $x, y \in X$ and by (22) and (23), we have

$$
\begin{gathered}
(a+1) f(a x+2 y)-(a+1) f(x+2 y) \\
-\left(a^{2}-1\right) f(x)=0,
\end{gathered}
$$

for all $x, y \in X$. Since $a \neq-1$, we have

$$
f(a x+2 y)-f(x+2 y)-(a-1) f(x)=0,
$$

for all $x, y \in X$ and letting $y=-y$ in (25), we have

$$
f(a x-2 y)-f(x-2 y)-(a-1) f(x)=0,
$$

for all $x, y \in X$. By (22) and (23), we have

$$
\begin{array}{r}
f(a x-2 y)+f(a x-2 y)-f(x+2 y) \\
-f(x-2 y)-2(a-1) f(x)=0,
\end{array}
$$

for all $x, y \in X$. Letting $y=2 y$ in (5), we have

$$
\begin{aligned}
& f(a x+2 y)+f(a x-2 y) \\
& \quad-a[f(x+2 y)+f(x-2 y)]=0,
\end{aligned}
$$

for all $x, y \in X$, and by (27) and (28), we have

$$
(a-1)[f(x+2 y)+f(x-2 y)-2 f(x)]=0,
$$

for all $x, y \in X$. Since $a \neq 1$, we have

$$
f(x+2 y)+f(x-2 y)-2 f(x)=0,
$$

for all $x, y \in X$, and hence $f$ is additive.

Combining Lemmas 4 and 5, we can get the following theorem.

Theorem 6. Let $f: X \rightarrow Y$ be an odd mapping with $f(0)=0$ satisfying (4). Then $f$ is an additive mapping.

Now if we assume that $f$ is an even function, (4) turns into the following equation with $b=1$ :

$$
\begin{aligned}
& f(a x+b y)+f(a x-b y)-2 a^{2} f(x)-2 b^{2} f(y) \\
& \quad-k[f(x+y)+f(x-y)-2 f(x)-2 f(y)]=0 .
\end{aligned}
$$

And in [21], the authors proved the following theorem.

Theorem 7 (see [21]). Let $f: X \rightarrow Y$ be a mapping with $f(0)=0$. Then $f$ is quadratic if and only if $f$ satisfies (31) for all $x \in X$, a fixed nonzero rational number $a$, and fixed real numbers $b, k$ with $a^{2} \neq b^{2}$.

By Theorems 6 and 7, we have the following theorem which is the conclusion of this section.

Theorem 8. Let $f: X \rightarrow Y$ be a mapping with $f(0)=0$. Then $f$ satisfies (4) if and only if $f$ is an additive-quadratic mapping. 


\section{The Generalized Hyers-Ulam Stability for (4)}

In this section, we prove the generalized Hyers-Ulam stability of functional equation (4) in fuzzy normed spaces. Throughout this section, we assume that $X$ is a linear space, $(Y, N)$ is a fuzzy Banach space, and $\left(Z, N^{\prime}\right)$ is a fuzzy normed space.

For any mapping $f: X \rightarrow Y$, we define the difference operator $D f: X^{2} \rightarrow Y$ by

$$
\begin{gathered}
D f(x, y)=f(a x+y)+f(a x-y)-a(a+1) f(x) \\
-a(a-1) f(-x)-[f(y)+f(-y)] \\
+k[f(x+y)+f(x-y) \\
-2 f(x)-f(y)-f(-y)],
\end{gathered}
$$

for all $x, y \in X$.

Theorem 9. Let $\phi: X^{2} \rightarrow Z$ be a function and let $r$ be a real number such that $0<|r|<|a|$ and

$$
N^{\prime}(\phi(a x, a y), t) \geq N^{\prime}(r \phi(x, y), t),
$$

for all $x, y \in X$ and all $t>0$. Let $f: X \rightarrow Y$ be an odd mapping such that $f(0)=0$ and

$$
N(D f(x, y), t) \geq N^{\prime}(\phi(x, y), t),
$$

for all $x, y \in X$ and all $t>0$. Then there exists a unique additive mapping $A: X \rightarrow Y$ such that the inequality

$$
N(f(x)-A(x), t) \geq N^{\prime}\left(\frac{\phi(x, 0)}{2(|a|-|r|)}, t\right)
$$

holds for all $x \in X$ and all $t>0$.

Proof. Since $f$ is an odd mapping, the inequality (34) is equivalent to the following inequality:

$$
\begin{aligned}
N( & f(a x+y)+f(a x-y)-2 a f(x) \\
& +k[f(x+y)+f(x-y)-2 f(x)], t) \\
& \geq N^{\prime}(\phi(x, y), t),
\end{aligned}
$$

for all $x, y \in X$ and all $t>0$. By (33) and (N3), we have

$$
\begin{aligned}
N^{\prime}\left(\phi\left(a^{n} x, a^{n} y\right), t\right) & \geq N^{\prime}\left(r^{n} \phi(x, y), t\right) \\
& =N^{\prime}\left(\phi(x, y), \frac{t}{|r|^{n}}\right),
\end{aligned}
$$

for all $x, y \in X$ and all $t>0$, and so by (37), we have

$$
N^{\prime}\left(\phi\left(a^{n} x, a^{n} y\right),|r|^{n} t\right) \geq N^{\prime}(\phi(x, y), t),
$$

for all $x, y \in X$ and all $t>0$. Letting $y=0$ in (36), by (N3), we have

$$
N\left(f(x)-\frac{f(a x)}{a}, \frac{t}{2|a|}\right) \geq N^{\prime}(\phi(x, 0), t),
$$

for all $x \in X$ and all $t>0$. By (38), (39), and (N3), we have

$$
\begin{aligned}
N\left(\frac{f\left(a^{n} x\right)}{a^{n}}-\frac{f\left(a^{n+1} x\right)}{a^{n+1}}, \frac{|r|^{n} t}{2|a|^{n+1}}\right) & \geq N^{\prime}\left(\phi\left(a^{n} x, 0\right),|r|^{n} t\right) \\
& \geq N^{\prime}(\phi(x, 0), t),
\end{aligned}
$$

for all $x \in X$, all $t>0$, and all positive integers $n$. Hence by (40) and (N4), for any $x \in X$, we have

$$
\begin{aligned}
& N\left(f(x)-\frac{f\left(a^{n} x\right)}{a^{n}}, \sum_{i=0}^{n-1} \frac{|r|^{i} t}{2|a|^{i+1}}\right) \\
& =N\left(\sum_{i=0}^{n-1}\left[\frac{f\left(a^{i} x\right)}{a^{i}}-\frac{f\left(a^{i+1} x\right)}{a^{i+1}}\right], \sum_{i=0}^{n-1} \frac{|r|^{i} t}{2|a|^{i+1}}\right) \\
& \geq \min \left\{N\left(\frac{f\left(a^{i} x\right)}{a^{i}}-\frac{f\left(a^{i+1} x\right)}{a^{i+1}}, \frac{|r|^{i} t}{2|a|^{i+1}}\right) \mid\right. \\
& \quad 0 \leq i \leq n-1\} \\
& \geq N^{\prime}(\phi(x, 0), t),
\end{aligned}
$$

for all $x \in X$, all $t>0$, and all positive integers $n$. So for any $x \in X$, we have

$$
\begin{aligned}
& N\left(\frac{f\left(a^{m} x\right)}{a^{m}}-\frac{f\left(a^{m+p} x\right)}{a^{m+p}}, \sum_{i=m}^{m+p-1} \frac{|r|^{i} t}{2|a|^{i+1}}\right) \\
& =N\left(\sum_{i=m}^{m+p-1}\left[\frac{f\left(a^{i} x\right)}{a^{i}}-\frac{f\left(a^{i+1} x\right)}{a^{i+1}}\right], \sum_{i=m}^{m+p-1} \frac{|r|^{i} t}{2|a|^{i+1}}\right) \\
& \geq \min \left\{N\left(\frac{f\left(a^{i} x\right)}{a^{i}}-\frac{f\left(a^{i+1} x\right)}{a^{i+1}}, \frac{|r|^{i} t}{2|a|^{i+1}}\right) \mid\right. \\
& \quad m \leq i \leq m+p-1\} \\
& \geq N^{\prime}(\phi(x, 0), t),
\end{aligned}
$$

for all $x \in X$, all $t>0$, all nonnegative integers $m$, and all positive integers $p$. Thus, by (42), for any $x \in X$, we have

$$
\begin{aligned}
& N\left(\frac{f\left(a^{m} x\right)}{a^{m}}-\frac{f\left(a^{m+p} x\right)}{a^{m+p}}, t\right) \\
& \quad \geq N^{\prime}\left(\phi(x, 0), \frac{t}{\sum_{i=m}^{m+p-1}\left(|r|^{i} / 2|a|^{i+1}\right)}\right),
\end{aligned}
$$

for all $x \in X$, all $t>0$, all nonnegative integers $m$, and all positive integers $p$. Since $\sum_{i=0}^{\infty}\left(|r|^{i} / 2|a|^{i+1}\right)$ is convergent, $\lim _{m \rightarrow \infty}\left(t / \sum_{i=m}^{m+p-1}\left(|r|^{i} / 2|a|^{i+1}\right)\right)=\infty$, and so by the usual 
argument $\left\{f\left(a^{n} x\right) / a^{n}\right\}$ is a Cauchy sequence in $(Y, N)$. Since $(Y, N)$ is a fuzzy Banach space, there is a mapping $A: X \rightarrow Y$ defined by

$$
\begin{aligned}
& A(x)=N-\lim _{n \rightarrow \infty} \frac{f\left(a^{n} x\right)}{a^{n}} \text { or } \\
& \lim _{n \rightarrow \infty} N\left(\frac{f\left(a^{n} x\right)}{a^{n}}-A(x), t\right)=1, \quad t>0,
\end{aligned}
$$

for all $x \in X$. Moreover by (41), we have

$$
\begin{aligned}
& N\left(f(x)-\frac{f\left(a^{n} x\right)}{a^{n}}, t\right) \\
& \quad \geq N^{\prime}\left(\phi(x, 0), \frac{t}{\sum_{i=0}^{n-1}\left(|r|^{i} / 2|a|^{i+1}\right)}\right),
\end{aligned}
$$

for all $x \in X$, all $t>0$, and all positive integers $n$. Let $\epsilon$ be a real number with $0<\epsilon<1$. Then, by (43), (44), (N4), and (N5), we have

$$
\begin{aligned}
& N(f(x)-A(x), t) \\
& \geq \min \left\{N\left(f(x)-\frac{f\left(a^{n} x\right)}{a^{n}},(1-\epsilon) t\right),\right. \\
& \left.\quad N\left(\frac{f\left(a^{n} x\right)}{a^{n}}-A(x), \epsilon t\right)\right\} \\
& \geq N^{\prime}\left(\phi(x, 0), \frac{(1-\epsilon) t}{\sum_{i=0}^{n-1}\left(|r|^{i} / 2|a|^{i+1}\right)}\right) \\
& \geq N^{\prime}(\phi(x, 0), 2(1-\epsilon)(|a|-|r|) t),
\end{aligned}
$$

for sufficiently large positive integer $n$, all $x \in X$, and all $t>0$ or $f(x)=N-\lim _{n \rightarrow \infty}\left(f\left(a^{n} x\right) / a^{n}\right)$. Since $N(x, \cdot)$ is continuous on $\mathbb{R}^{+}$for all $x$ from (N2) and (N6), by taking $\epsilon \rightarrow 0$, we get

$$
N(f(x)-A(x), t) \geq N^{\prime}(\phi(x, 0), 2(|a|-|r|) t),
$$

for all $x \in X$ and all $t>0$, and so we have (35).

By (33), (34), and (N3), we have

$$
\begin{aligned}
N\left(\frac{D f\left(a^{n} x, a^{n} y\right)}{a^{n}}, t\right) & \geq N^{\prime}\left(\phi\left(a^{n} x, a^{n} y\right),|a|^{n} t\right) \\
& \geq N^{\prime}\left(\phi(x, y), \frac{|a|^{n}}{|r|^{n}} t\right),
\end{aligned}
$$

for all $x, y \in X$ and all $t>0$. Since $\lim _{n \rightarrow \infty} N^{\prime}(\phi(x, y)$, $\left.\left(|a|^{n} /|r|^{n}\right) t\right)=1$ and

$$
\lim _{n \rightarrow \infty} N\left(D A(x, y)-\frac{D f\left(a^{n} x, a^{n} y\right)}{a^{n}}, t\right)=1,
$$

for all $x \in X$ and all $t>0$, by (44), (48), and (N4), we have

$$
\begin{aligned}
& N(D A(x, y), t) \\
& \geq \min \left\{N\left(D A(x, y)-\frac{D f\left(a^{n} x, a^{n} y\right)}{a^{n}}, \frac{t}{2}\right),\right. \\
& \left.\quad N\left(\frac{D f\left(a^{n} x, a^{n} y\right)}{a^{n}}, \frac{t}{2}\right)\right\} \\
& \geq N\left(\frac{D f\left(a^{n} x, a^{n} y\right)}{a^{n}}, \frac{t}{2}\right) \\
& \geq N^{\prime}\left(\phi(x, y), \frac{|a|^{n}}{2|r|^{n}} t\right),
\end{aligned}
$$

for sufficiently large $n$, all $x, y \in X$, and all $t>0$ or $N-\lim \left(D f\left(a^{n} x, a^{n} y\right) / a^{n}\right)=0$. Since $\lim _{n \rightarrow \infty} N^{\prime}(\phi(x, y)$, $\left.\left(|a|^{n} /|r|^{n}\right) t\right)=1, N(D A(x, y), t)=1$ for all $t>0$, and so, by (N2), $D A(x, y)=0$ for all $x, y \in X$. By Theorem $8, A$ is additive.

To prove the uniqueness of $A$, let $A_{1}: X \rightarrow Y$ be another additive mapping satisfying (35). Then for any $x \in X$ and a positive integer $n, A_{1}\left(a^{n} x\right)=a^{n} A_{1}(x)$, and so by (45),

$$
\begin{aligned}
& N\left(A(x)-A_{1}(x), t\right) \\
& \geq \min \left\{N\left(\frac{A\left(a^{n} x\right)}{a^{n}}-\frac{f\left(a^{n} x\right)}{a^{n}}, \frac{t}{2}\right),\right. \\
& \left.\quad N\left(\frac{f\left(a^{n} x\right)}{a^{n}}-\frac{A_{1}\left(a^{n} x\right)}{a^{n}}, \frac{t}{2}\right)\right\} \\
& \geq N^{\prime}\left(\phi\left(a^{n} x, 0\right), a^{n}(|a|-|r|) t\right) \\
& \geq N^{\prime}\left(\phi(x, 0), \frac{a^{n}(|a|-|r|) t}{|r|^{n}}\right)
\end{aligned}
$$

holds for all $x \in X$, all positive integers $n$, and all $t>0$. Since $|r|<|a|, \lim _{n \rightarrow \infty} N^{\prime}\left(\phi(x, 0),\left(a^{n}(|a|-|r|) t\right) /|r|^{n}\right)=1$, and so $A(x)=A_{1}(x)$ for all $x \in X$.

Now we deal with the even function case.

Theorem 10. Let $\phi: X^{2} \rightarrow Z$ be a function and let $r$ be a real number such that $0<|r|<a^{2}$ and

$$
N^{\prime}(\phi(a x, a y), t) \geq N^{\prime}(r \phi(x, y), t),
$$

for all $x, y \in X$ and all $t>0$. Let $f: X \rightarrow Y$ be an even mapping satisfying $f(0)=0$ and (34). Then there exists a unique quadratic mapping $Q: X \rightarrow Y$ such that the inequality

$$
N(f(x)-Q(x), t) \geq N^{\prime}\left(\frac{1}{2\left(a^{2}-|r|\right)} \phi(x, 0), t\right)
$$

holds for all $x \in X$ and all $t>0$. 
Proof. Since $f$ is an even mapping, the inequality (34) is equivalent to the following inequality:

$$
\begin{aligned}
N( & f(a x+y)+f(a x-y)-2 a^{2} f(x)-2 f(y) \\
& +k[f(x+y)+f(x-y)-2 f(x)-2 f(y)], t) \\
\geq & N^{\prime}(\phi(x, y), t),
\end{aligned}
$$

for all $x, y \in X$ and all $t>0$. By (52) and (N3), we have

$$
\begin{aligned}
N^{\prime}\left(\phi\left(a^{n} x, a^{n} y\right), t\right) & \geq N^{\prime}\left(r^{n} \phi(x, y), t\right) \\
& =N^{\prime}\left(\phi(x, y), \frac{t}{|r|^{n}}\right),
\end{aligned}
$$

for all $x, y \in X$ and all $t>0$, and so by (55), we have

$$
N^{\prime}\left(\phi\left(a^{n} x, a^{n} y\right),|r|^{n} t\right) \geq N^{\prime}(\phi(x, y), t)
$$

for all $x, y \in X$ and all $t>0$. Letting $y=0$ in (54), by (N3), we have

$$
N\left(f(x)-\frac{f(a x)}{a^{2}}, \frac{t}{2 a^{2}}\right) \geq N^{\prime}(\phi(x, 0), t),
$$

for all $x \in X$ and all $t>0$. By (52), (56), (57), and (N3), we have

$$
\begin{aligned}
N\left(\frac{f\left(a^{n} x\right)}{a^{2 n}}-\frac{f\left(a^{n+1} x\right)}{a^{2(n+1)}}, \frac{|r|^{n} t}{2 a^{2(n+1)}}\right) & \geq N^{\prime}\left(\phi\left(a^{n} x, 0\right),|r|^{n} t\right) \\
& \geq N^{\prime}(\phi(x, 0), t),
\end{aligned}
$$

for all $x \in X$, all $t>0$, and all positive integers $n$. Hence by (58) and (N4), for any $x \in X$, we have

$$
\begin{aligned}
& N\left(f(x)-\frac{f\left(a^{n} x\right)}{a^{2 n}}, \sum_{i=0}^{n-1} \frac{|r|^{i} t}{2 a^{2(i+1)}}\right) \\
& =N\left(\sum_{i=0}^{n-1}\left[\frac{f\left(a^{i} x\right)}{a^{2 i}}-\frac{f\left(a^{i+1} x\right)}{a^{2(i+1)}}\right], \sum_{i=0}^{n-1} \frac{|r|^{i} t}{2 a^{2(i+1)}}\right) \\
& \geq \min \left\{N\left(\frac{f\left(a^{i} x\right)}{a^{2 i}}-\frac{f\left(a^{i+1} x\right)}{a^{2(i+1)}}, \frac{|r|^{i} t}{2 a^{2(i+1)}}\right) \mid\right. \\
& \quad 0 \leq i \leq n-1\} \\
& \geq N^{\prime}(\phi(x, 0), t),
\end{aligned}
$$

for all $x \in X$, all $t>0$, and all positive integers $n$. So for any $x \in X$, we have

$$
\begin{aligned}
& N\left(\frac{f\left(a^{m} x\right)}{a^{2 m}}-\frac{f\left(a^{m+p} x\right)}{a^{2(m+p)}}, \sum_{i=m}^{m+p-1} \frac{|r|^{i} t}{2 a^{2(i+1)}}\right) \\
& =N\left(\sum_{i=m}^{m+p-1}\left[\frac{f\left(a^{i} x\right)}{a^{2 i}}-\frac{f\left(a^{i+1} x\right)}{a^{2(i+1)}}\right], \sum_{i=m}^{m+p-1} \frac{|r|^{i} t}{2 a^{2(i+1)}}\right) \\
& \geq \min \left\{N\left(\frac{f\left(a^{i} x\right)}{a^{2 i}}-\frac{f\left(a^{i+1} x\right)}{a^{2(i+1)}}, \frac{|r|^{i} t}{2 a^{2(i+1)}}\right) \mid\right. \\
& \quad m \leq i \leq m+p-1\} \\
& \geq N^{\prime}(\phi(x, 0), t),
\end{aligned}
$$

for all $x \in X$, all $t>0$, all nonnegative integers $m$, and all positive integers $p$. Thus, by (60) and (N3), for any $x \in X$, we have

$$
\begin{aligned}
& N\left(\frac{f\left(a^{m} x\right)}{a^{2 m}}-\frac{f\left(a^{m+p} x\right)}{\left.a^{2(m+p)}, t\right)}\right. \\
& \quad \geq N^{\prime}\left(\phi(x, 0), \frac{t}{\sum_{i=m}^{m+p-1}\left(|r|^{i} / 2 a^{2(i+1)}\right)}\right),
\end{aligned}
$$

for all $x \in X$, all $t>0$, all nonnegative integers $m$, and all positive integers $p$. Since $\sum_{i=0}^{\infty}\left(|r|^{i} / 2 a^{2(i+1)}\right)$ is convergent, $\lim _{m \rightarrow \infty}\left(t / \sum_{i=m}^{m+p-1}\left(|r|^{i} / 2 a^{2(i+1)}\right)\right)=\infty$, and so by the usual argument again, $\left\{f\left(a^{n} x\right) / a^{2 n}\right\}$ is a Cauchy sequence in $(Y, N)$. Since $(Y, N)$ is a fuzzy Banach space, there is a mapping $Q$ : $X \rightarrow Y$ defined by

$$
\begin{aligned}
& Q(x)=N-\lim _{n \rightarrow \infty} \frac{f\left(a^{n} x\right)}{a^{2 n}} \text { or } \\
& \lim _{n \rightarrow \infty} N\left(\frac{f\left(a^{n} x\right)}{a^{2 n}}-Q(x), t\right)=1, \quad t>0,
\end{aligned}
$$

for all $x \in X$. Moreover by (59), we have

$$
\begin{aligned}
& N\left(f(x)-\frac{f\left(a^{n} x\right)}{a^{2 n}}, t\right) \\
& \quad \geq N^{\prime}\left(\phi(x, 0), \frac{t}{\sum_{i=0}^{n-1}\left(|r|^{i} / 2 a^{2(i+1)}\right)}\right),
\end{aligned}
$$


for all $x \in X$, all $t>0$, and all positive integers $n$. Let $\epsilon$ be a real number with $0<\epsilon<1$. Then, by (62), (63), and (N4), we have

$$
\begin{gathered}
N(f(x)-Q(x), t) \\
\geq \min \left\{N\left(f(x)-\frac{f\left(a^{n} x\right)}{a^{2 n}},(1-\epsilon) t\right),\right. \\
\left.N\left(\frac{f\left(a^{n} x\right)}{a^{2 n}}-Q(x), \epsilon t\right)\right\} \\
\geq N^{\prime}\left(\phi(x, 0), \frac{(1-\epsilon) t}{\sum_{i=0}^{n-1}\left(|r|^{i} / 2 a^{2(i+1)}\right)}\right) \\
\geq N^{\prime}\left(\phi(x, 0), 2(1-\epsilon)\left(a^{2}-|r|\right) t\right),
\end{gathered}
$$

for sufficiently large positive integer $n$, all $x \in X$, and all $t>0$ or $f(x)=N-\lim _{n \rightarrow \infty}\left(f\left(a^{n} x\right) / a^{2 n}\right)$. Since $N(x, \cdot)$ is continuous on $\mathbb{R}^{+}$for all $x$ from (N2) and (N6), we get

$$
N(f(x)-Q(x), t) \geq N^{\prime}\left(\phi(x, 0), 2\left(a^{2}-|r|\right) t\right),
$$

for all $x \in X$ and all $t>0$, and so we have (53). By (34) and (N5), we have

$$
\begin{aligned}
N\left(\frac{D f\left(a^{n} x, a^{n} y\right)}{a^{2 n}}, t\right) & \geq N^{\prime}\left(\phi\left(a^{n} x, a^{n} y\right), a^{2 n} t\right) \\
& \geq N^{\prime}\left(\phi(x, y), \frac{a^{2 n}}{|r|^{n}} t\right),
\end{aligned}
$$

for all $x, y \in X$ and all $t>0$. Since

$$
\lim _{n \rightarrow \infty} N\left(D Q(x, y)-\frac{D f\left(a^{n} x, a^{n} y\right)}{a^{2 n}}, t\right)=1,
$$

for all $x \in X$ and all $t>0$, and by (62), (66), and (N4), we have

$$
\begin{aligned}
& N(D Q(x, y), t) \\
& \geq \min \left\{N\left(D Q(x, y)-\frac{D f\left(a^{n} x, a^{n} y\right)}{a^{2 n}}, \frac{t}{2}\right),\right. \\
& \left.N\left(\frac{D f\left(a^{n} x, a^{n} y\right)}{a^{2 n}}, \frac{t}{2}\right)\right\} \\
& \geq N\left(\frac{D f\left(a^{n} x, a^{n} y\right)}{a^{2 n}}, \frac{t}{2}\right) \\
& \geq N^{\prime}\left(\phi(x, y), \frac{a^{2 n}}{2|r|^{n}} t\right),
\end{aligned}
$$

for sufficiently large $n$, all $x, y \in X$, and all $t>0$ or $N-$ $\lim \left(D f\left(a^{n} x, a^{n} y\right) / a^{2 n}\right)=0$.

Since $\lim _{n \rightarrow \infty} N^{\prime}\left(\phi(x, y),\left(a^{2 n} /|r|^{n}\right) t\right)=1, N(D Q(x, y)$, $t)=1$ for all $t>0$, and so by (N2), $D Q(x, y)=0$ for all $x, y \in X$. By Theorem $8, Q$ is quadratic.
To prove the uniqueness of $Q$, let $Q_{1}: X \rightarrow Y$ be another quadratic mapping satisfying (53). Then for any $x \in X$ and a positive integer $n, Q_{1}\left(a^{n} x\right)=a^{2 n} Q_{1}(x)$, and so by (63),

$$
\begin{aligned}
& N\left(Q(x)-Q_{1}(x), t\right) \\
& \geq \min \left\{N\left(\frac{Q\left(a^{n} x\right)}{a^{2 n}}-\frac{f\left(a^{n} x\right)}{a^{2 n}}, \frac{t}{2}\right),\right. \\
& \left.N\left(\frac{Q_{1}\left(a^{n} x\right)}{a^{2 n}}-\frac{f\left(a^{n} x\right)}{a^{2 n}}, \frac{t}{2}\right)\right\} \\
& \geq N^{\prime}\left(\phi\left(a^{n} x, 0\right), a^{2 n}\left(a^{2}-|r|\right) t\right) \\
& \geq N^{\prime}\left(\phi(x, 0), \frac{a^{2 n}\left(a^{2}-|r|\right) t}{|r|^{n}}\right)
\end{aligned}
$$

holds for all $x \in X$, all positive integers $n$, and all $t>0$. Since $|r|<a^{2}, \lim _{n \rightarrow \infty} N^{\prime}\left(\phi(x, 0),\left(a^{2 n}\left(a^{2}-|r|\right) t\right) /|r|^{n}\right)=1$, and so $Q(x)=Q_{1}(x)$ for all $x \in X$.

Now we consider the next two theorems which are similar to Theorems 9 and 10. The proofs are straightforward and similar to those of Theorems 9 and 10.

Theorem 11. Let $\phi: X^{2} \rightarrow Z$ be a function and let $r$ be a real number such that $|a|<|r|$ such that

$$
N^{\prime}\left(\phi\left(\frac{x}{a}, \frac{y}{a}\right), t\right) \geq N^{\prime}\left(\frac{1}{r} \phi(x, y), t\right),
$$

for all $x, y \in X$ and all $t>0$. Let $f: X \rightarrow Y$ be an odd mapping with $f(0)=0$ satisfying (34). Then there exists a unique additive mapping $A: X \rightarrow Y$ such that the inequality

$$
N(A(x)-f(x), t) \geq N^{\prime}\left(\frac{\operatorname{ar} \phi(x, 0)}{2(|a|-|r|)}, t\right)
$$

holds for all $x \in X$ and all $t>0$.

Theorem 12. Let $\phi: X^{2} \rightarrow Z$ be a function and let $r$ be a real number such that $a^{2}<|r|$ such that

$$
N^{\prime}\left(\phi\left(\frac{x}{a}, \frac{y}{a}\right), t\right) \geq N^{\prime}\left(\frac{1}{r} \phi(x, y), t\right),
$$

for all $x, y \in X$ and all $t>0$. Let $f: X \rightarrow Y$ be an even mapping with $f(0)=0$ satisfying (34). Then there exists a unique additive mapping $Q: X \rightarrow Y$ such that the inequality

$$
N(Q(x)-f(x), t) \geq N^{\prime}\left(\frac{a^{2} r \phi(x, 0)}{2\left(a^{2}-|r|\right)}, t\right)
$$

holds for all $x \in X$ and all $t>0$.

By combining Theorems 9 and 10, we can have the following theorem which is the main theorem of the paper.

Theorem 13. Let $\phi: X^{2} \rightarrow Z$ be a function and let $r$ be a real number such that $|r|<\min \left\{|a|, a^{2}\right\}$ such that

$$
N^{\prime}(\phi(a x, a y), t) \geq N^{\prime}(r \phi(x, y), t),
$$


for all $x, y \in X$ and all $t>0$. Let $f: X \rightarrow Y$ be $a$ mapping with $f(0)=0$ satisfying (34). Then there exists a unique additive-quadratic mapping $F: X \rightarrow Y$ such that the inequality

$$
N(F(x)-f(x), t) \geq N_{1}(x, t)
$$

holds for all $x \in X$ and all $t>0$, where

$$
\begin{aligned}
& N_{1}(x, t) \\
& =\min \left\{N^{\prime}\left(\frac{\phi(x, 0)}{2(|a|-|r|)}, t\right), N^{\prime}\left(\frac{\phi(-x, 0)}{2(|a|-|r|)}, t\right),\right. \\
& \left.N^{\prime}\left(\frac{\phi(x, 0)}{2\left(a^{2}-|r|\right)}, t\right), N^{\prime}\left(\frac{\phi(-x, 0)}{2\left(a^{2}-|r|\right)}, t\right)\right\} .
\end{aligned}
$$

Proof. By (34), we have

$$
\begin{aligned}
N & \left(D f_{o}(x, y), t\right) \\
& \geq \min \left\{N^{\prime}(\phi(x, y), t), N^{\prime}(\phi(-x,-y), t)\right\},
\end{aligned}
$$

for all $x, y \in X$ and $t>0$. By Theorem 9 , there is a unique additive mapping $A: X \rightarrow Y$ such that

$$
\begin{array}{r}
N\left(f_{o}(x)-A(x), t\right) \\
\geq \min \left\{N^{\prime}\left(\frac{\phi(x, 0)}{2(|a|-|r|)}, t\right), N^{\prime}\left(\frac{\phi(-x, 0)}{2(|a|-|r|)}, t\right),\right. \\
\left.\quad N^{\prime}\left(\frac{\operatorname{ar} \phi(x, 0)}{2(|a|-|r|)}, t\right), N^{\prime}\left(\frac{\operatorname{ar} \phi(-x, 0)}{2(|a|-|r|)}, t\right)\right\}
\end{array}
$$

holds for all $x \in X$ and all $t>0$.

By (34), we have

$$
\begin{aligned}
N & \left(D f_{e}(x, y), t\right) \\
& \geq \min \left\{N^{\prime}(\phi(x, y), t), N^{\prime}(\phi(-x,-y), t)\right\},
\end{aligned}
$$

for all $x, y \in X$ and $t>0$. By Theorem 10, there is a unique quadratic mapping $Q: X \rightarrow Y$ such that

$$
\begin{aligned}
& N\left(f_{e}(x)-Q(x), t\right) \\
& \geq \min \left\{N^{\prime}\left(\frac{\phi(x, 0)}{2\left(a^{2}-|r|\right)}, t\right), N^{\prime}\left(\frac{\phi(-x, 0)}{2\left(a^{2}-|r|\right)}, t\right),\right. \\
& \left.N^{\prime}\left(\frac{a^{2} r \phi(x, 0)}{2\left(a^{2}-|r|\right)}, t\right), N^{\prime}\left(\frac{a^{2} r \phi(-x, 0)}{2\left(a^{2}-|r|\right)}, t\right)\right\}
\end{aligned}
$$

holds for all $x \in X$ and all $t>0$. Let $F(x)=A(x)+Q(x)$. Then by (78) and (80), we have (75).

The uniqueness of $F$ satisfying (75) is trivial.

Also, if we combine Theorems 11 and 12, we have the following theorem.
Theorem 14. Let $\phi: X^{2} \rightarrow Z$ be a function and let $r$ be a real number such that $|r|>\max \left\{|a|, a^{2}\right\}$ such that

$$
N^{\prime}\left(\phi\left(\frac{x}{a}, \frac{y}{a}\right), t\right) \geq N^{\prime}\left(\frac{1}{r} \phi(x, y), t\right)
$$

for all $x, y \in X$ and all $t>0$. Let $f: X \rightarrow Y$ be $a$ mapping with $f(0)=0$ satisfying (34). Then there exists a unique additive-quadratic mapping $F: X \rightarrow Y$ such that the inequality

$$
N(F(x)-f(x), t) \geq N_{2}(x, t)
$$

holds for all $x \in X$ and all $t>0$, where

$$
\begin{aligned}
& N_{2}(x, t) \\
& =\min \left\{N^{\prime}\left(\frac{\operatorname{ar} \phi(x, 0)}{2(|a|-|r|)}, t\right), N^{\prime}\left(\frac{\operatorname{ar} \phi(-x, 0)}{2(|a|-|r|)}, t\right),\right. \\
& \left.N^{\prime}\left(\frac{a^{2} r \phi(x, 0)}{2\left(a^{2}-|r|\right)}, t\right), N^{\prime}\left(\frac{a^{2} r \phi(-x, 0)}{2\left(a^{2}-|r|\right)}, t\right)\right\} .
\end{aligned}
$$

Among the examples of the function $\phi(x, y)$, there are lots of meaningful ones satisfying $\phi(c x, c y)=k(c) \phi(x, y)$ for all real numbers $c$ and for some real number $k(c)$. The following theorem says that a strong and useful result can be obtained in such cases.

Theorem 15. Let $\phi: X^{2} \rightarrow Z$ be a function and let $r$ be a real number such that $|r| \neq a^{2},|a|$ such that

$$
N^{\prime}(\phi(a x, a y), t)=N^{\prime}(r \phi(x, y), t)
$$

for all $x, y \in X$ and all $t>0$. Let $f: X \rightarrow Y$ be $a$ mapping with $f(0)=0$ satisfying (34). Then there exists a unique additive-quadratic mapping $F: X \rightarrow Y$ such that the inequality

$$
N(F(x)-f(x), t) \geq N_{3}(x, t)
$$

holds for all $x \in X$ and all $t>0$, where

$$
\begin{aligned}
& N_{3}(x, t) \\
& =\min \left\{N^{\prime}\left(\frac{\phi(x, 0)}{2(|a|-|r|)}, t\right), N^{\prime}\left(\frac{\phi(-x, 0)}{2(|a|-|r|)}, t\right),\right. \\
& N^{\prime}\left(\frac{\phi(x, 0)}{2\left(a^{2}-|r|\right)}, t\right), N^{\prime}\left(\frac{\phi(-x, 0)}{2\left(a^{2}-|r|\right)}, t\right), \\
& N^{\prime}\left(\frac{\operatorname{ar} \phi(x, 0)}{2(|a|-|r|)}, t\right), N^{\prime}\left(\frac{\operatorname{ar} \phi(-x, 0)}{2(|a|-|r|)}, t\right), \\
& \left.N^{\prime}\left(\frac{a^{2} r \phi(x, 0)}{2\left(a^{2}-|r|\right)}, t\right), N^{\prime}\left(\frac{a^{2} r \phi(-x, 0)}{2\left(a^{2}-|r|\right)}, t\right)\right\} .
\end{aligned}
$$

We can use Theorem 15 to get a classical result in the framework of normed spaces. For example, it is well known 
that for any normed space $(X,\|\cdot\|)$, mappings $N_{X}, N_{X}^{\prime}$ : $X \times \mathbb{R} \rightarrow[0,1]$, defined by

$$
\begin{gathered}
N_{X}(x, t)= \begin{cases}0, & \text { if } t \leq 0, \\
\frac{t}{t+\|x\|}, & \text { if } t>0,\end{cases} \\
N_{X}^{\prime}(x, t)= \begin{cases}0, & \text { if } t<\|x\|, \\
1, & \text { if } t \geq\|x\|,\end{cases}
\end{gathered}
$$

are fuzzy norms on $\mathrm{X}$. In [12, 13, 22], some examples are provided for the fuzzy norms $N_{X}, N_{X}^{\prime}$ and other fuzzy norms. Here especially using the fuzzy norm $N_{X}$ and taking $\phi(x, y)=$ $\|x\|^{p}\|y\|^{p}+\|x\|^{2 p}+\|y\|^{2 p}$, we have the following example.

Example 16. Let $f: X \rightarrow Y$ be a mapping such that $f(0)=0$ and

$$
\|D f(x, y)\| \leq\|x\|^{p}\|y\|^{p}+\|x\|^{2 p}+\|y\|^{2 p},
$$

for all $x, y \in X$, a fixed rational number $a \neq-1,0,1$, and a fixed positive number $p$ such that $p \neq 1 / 2,1$. Then there exists a unique additive-quadratic mapping $F: X \rightarrow Y$ such that the inequality

$$
\begin{aligned}
\|F(x)-f(x)\| \\
\leq \min \left\{\frac{\|x\|^{2 p}}{2\left|a^{2 p}-\right| a||}, \frac{a^{2 p+1}\|x\|^{2 p}}{2\left|a^{2 p}-\right| a||},\right. \\
\left.\frac{\|x\|^{2 p}}{2\left|a^{2 p}-a^{2}\right|}, \frac{a^{2 p+2}\|x\|^{2 p}}{2\left|a^{2 p}-a^{2}\right|}\right\}
\end{aligned}
$$

holds for all $x \in X$.

The condition $p \neq 1 / 2,1$ in Example 16 is indispensable. The following example shows that the inequality (88) is not stable for $p=1 / 2,1$, especially in the case of $a=2$. We will give the proof when $p=1 / 2$, and the proof when $p=1$ is similar.

Example 17. Let $p$ be a real number with $p=1 / 2$. Define mappings $t, s: \mathbb{R} \rightarrow \mathbb{R}$ by

$$
\begin{aligned}
& t(x)= \begin{cases}x, & \text { if }|x|<1, \\
-1, & \text { if } x \leq-1, \\
1, & \text { if } 1 \leq x,\end{cases} \\
& s(x)= \begin{cases}x^{2}, & \text { if }|x|<1, \\
1, & \text { ortherwise }\end{cases}
\end{aligned}
$$

and a mapping $f: \mathbb{R} \rightarrow \mathbb{R}$ by

$$
f(x)=\sum_{n=0}^{\infty}\left[\frac{t\left(2^{n} x\right)}{2^{n}}+\frac{s\left(2^{n} x\right)}{4^{n}}\right] .
$$

We will show that $f$ satisfies the following inequality:

$$
\left|D_{2} f(x, y)\right| \leq \frac{200}{3}\left(|x|^{1 / 2}|y|^{1 / 2}+|x|+|y|\right),
$$

for all $x, y \in \mathbb{R}$. Here,

$$
\begin{aligned}
D_{2} f(x, y)= & f(2 x+y)+f(2 x-y) \\
& -4 f(x)-2 f(-x)-f(x+y)-f(x-y) .
\end{aligned}
$$

But there do not exist an additive-quadratic mapping $F$ : $\mathbb{R} \rightarrow \mathbb{R}$ and a nonnegative constant $K$ such that

$$
|F(x)-f(x)| \leq K|x|,
$$

for all $x \in \mathbb{R}$.

Proof. Note that $\left|f_{o}(x)\right| \leq 2, s_{o}(x)=0$, and $t_{o}(x)=t(x)$ for all $x \in \mathbb{R}$. First, suppose that $(1 / 2) \leq|x|^{1 / 2}|y|^{1 / 2}+|x|+|y|$. Then $\left|D_{2} f_{o}(x, y)\right| \leq 40\left(|x|^{1 / 2}|y|^{1 / 2}+|x|+|y|\right)$. Now suppose that $(1 / 2)>|x|^{1 / 2}|y|^{1 / 2}+|x|+|y|$. Then there is a positive integer $m$ such that

$$
\frac{1}{2^{m+2}} \leq|x|^{1 / 2}|y|^{1 / 2}+|x|+|y|<\frac{1}{2^{m+1}}
$$

and so

$$
2^{m}|x|<\frac{1}{2}, \quad 2^{m}|y|<\frac{1}{2} .
$$

Hence, we have

$$
\left\{2^{m-1}(2 x \pm y), 2^{m-1}(x \pm y), 2^{m-1} x, 2^{m-1} y\right\} \subseteq(-1,1),
$$

and so for any $n=0,1,2, \ldots, m-1$,

$$
D_{2} t_{0}\left(2^{n} x, 2^{n} y\right)=0,
$$

for all $x, y \in X$. Thus,

$$
\begin{aligned}
D_{2} f_{o}(x, y) & =\sum_{n=0}^{\infty} \frac{1}{2^{n}} D_{2} t\left(2^{n} x, 2^{n} y\right) \\
& =\sum_{n=m}^{\infty} \frac{1}{2^{n}} D_{2} t\left(2^{n} x, 2^{n} y\right) \\
& \leq \frac{1}{2^{m-1}} \leq 8\left(|x|^{1 / 2}|y|^{1 / 2}+|x|+|y|\right) .
\end{aligned}
$$

Note that $\left|f_{e}(x)\right| \leq 4 / 3$ for all $x \in \mathbb{R}, t_{e}(x)=0$, and $s_{e}(x)=s(x)$. First, suppose that $(1 / 2) \leq|x|^{1 / 2}|y|^{1 / 2}+|x|+|y|$. Then $\left|D_{2} f_{e}(x, y)\right| \leq(80 / 3)\left(|x|^{1 / 2}|y|^{1 / 2}+|x|+|y|\right)$ for all $x, y \in$ $\mathbb{R}$. Now suppose that $(1 / 2)>|x|^{1 / 2}|y|^{1 / 2}+|x|+|y|$. Then there is a positive integer $k$ such that

$$
\frac{1}{2^{2 k+4}} \leq|x|^{1 / 2}|y|^{1 / 2}+|x|+|y|<\frac{1}{2^{2 k+2}},
$$

and so

$$
2^{k}|x|<\frac{1}{2}, \quad 2^{k}|y|<\frac{1}{2} .
$$


Hence, we have

$$
\begin{aligned}
& \left\{2^{k-1}(2 x \pm y), 2^{k-1}(x \pm y), 2^{k-1} x, 2^{k-1} y\right\} \\
& \subseteq(-1,1),
\end{aligned}
$$

and so for any $n=0,1,2, \ldots, k-1$,

$$
D_{2} s_{e}\left(2^{n} x, 2^{n} y\right)=0,
$$

for all $x, y \in X$. Thus,

$$
\begin{aligned}
D_{2} f_{e}(x, y) & =\sum_{n=0}^{\infty} \frac{1}{4^{n}} D_{2} s_{e}\left(2^{n} x, 2^{n} y\right) \\
& =\sum_{n=k}^{\infty} \frac{1}{4^{n}} D_{2} s_{e}\left(2^{n} x, 2^{n} y\right) \\
& \leq \frac{64}{3}\left(|x|^{1 / 2}|y|^{1 / 2}+|x|+|y|\right) .
\end{aligned}
$$

Hence, we have

$$
\begin{aligned}
& \left|D_{2} f_{o}(x, y)\right| \leq 40\left(|x|^{1 / 2}|y|^{1 / 2}+|x|+|y|\right), \\
& \left|D_{2} f_{e}(x, y)\right| \leq \frac{80}{3}\left(|x|^{1 / 2}|y|^{1 / 2}+|x|+|y|\right),
\end{aligned}
$$

for all $x, y \in X$, and so we have (92).

Suppose that there exist an additive mapping $A: \mathbb{R} \rightarrow$ $\mathbb{R}$, a quadratic mapping $Q: \mathbb{R} \rightarrow \mathbb{R}$, and a nonnegative constant $K$ such that $A+Q$ satisfies (94). Since $|f(x)| \leq$ (10/3), by (94), we have

$$
-\frac{10}{3 n}-K \frac{|x|}{n} \leq \frac{A(x)}{n}+Q(x) \leq \frac{10}{3 n}+K \frac{|x|}{n},
$$

for all $x \in X$ and all positive integers $n$, and so $Q(x)=0$ for all $x \in X$. Since $A$ is additive,

$$
\left|f_{e}(x)\right| \leq K|x|
$$

for all $x \in X$. Take a positive integer $l$ such that $l>M$, and pick $x \in \mathbb{R}$ with $0<2^{l} x<1$. Then

$$
f_{e}(x)=\sum_{n=0}^{\infty} \frac{s\left(2^{n} x\right)}{4^{n}} \geq \sum_{n=0}^{l-1} \frac{s\left(2^{n} x\right)}{4^{n}} \geq l x^{2}>M x^{2},
$$

which is in contradiction with (107).

\section{Conflict of Interests}

The authors declare that there is no conflict of interests regarding the publication of this paper.

\section{References}

[1] S. M. Ulam, A Collection of Mathematical Problems, Interscience Tracts in Pure and Applied Mathematics, no. 8, Interscience Publishers, New York, NY, USA, 1960.
[2] D. H. Hyers, "On the stability of the linear functional equation," Proceedings of the National Academy of Sciences of the United States of America, vol. 27, pp. 222-224, 1941.

[3] T. Aoki, "On the stability of the linear transformation in Banach spaces," Journal of the Mathematical Society of Japan, vol. 2, pp. 64-66, 1950.

[4] M. S. Moslehian and T. M. Rassias, "Stability of functional equations in non-Archimedean spaces," Applicable Analysis and Discrete Mathematics, vol. 1, no. 2, pp. 325-334, 2007.

[5] Th. M. Rassias, "On the stability of the linear mapping in Banach spaces," Proceedings of the American Mathematical Society, vol. 72, no. 2, pp. 297-300, 1978.

[6] P. W. Cholewa, "Remarks on the stability of functional equations," Aequationes Mathematicae, vol. 27, no. 1-2, pp. 76-86, 1984.

[7] K. Cieplinski, "Applications of fixed point theorems to the Hyers-Ulam stability of functional equations-a survey," Annals of Functional Analysis, vol. 3, no. 1, pp. 151-164, 2012.

[8] St. Czerwik, "On the stability of the quadratic mapping in normed spaces," Abhandlungen aus dem Mathematischen Seminar der Universität Hamburg, vol. 62, pp. 59-64, 1992.

[9] P. Găvruta, "A generalization of the Hyers-Ulam-Rassias stability of approximately additive mappings," Journal of Mathematical Analysis and Applications, vol. 184, no. 3, pp. 431-436, 1994.

[10] M. Mirzavaziri and M. S. Moslehian, "A fixed point approach to stability of a quadratic equation," Bulletin of the Brazilian Mathematical Society, vol. 37, no. 3, pp. 361-376, 2006.

[11] H.-M. Kim, J. M. Rassias, and J. Lee, "Fuzzy approximation of Euler-Lagrange quadratic mappings," Journal of Inequalities and Applications, vol. 2013, article 358, pp. 1-15, 2013.

[12] A. K. Mirmostafaee and M. S. Moslehian, "Fuzzy almost quadratic functions," Results in Mathematics, vol. 52, no. 1-2, pp. 161-177, 2008.

[13] A. K. Mirmostafaee and M. S. Moslehian, "Fuzzy versions of Hyers-Ulam-Rassias theorem," Fuzzy Sets and Systems, vol. 159, no. 6, pp. 720-729, 2008.

[14] A. K. Katsaras, "Fuzzy topological vector spaces. II," Fuzzy Sets and Systems, vol. 12, no. 2, pp. 143-154, 1984.

[15] S. C. Cheng and J. N. Mordeson, "Fuzzy linear operators and fuzzy normed linear spaces," Bulletin of the Calcutta Mathematical Society, vol. 86, no. 5, pp. 429-436, 1994.

[16] I. Kramosil and J. Michálek, "Fuzzy metrics and statistical metric spaces," Kybernetika, vol. 11, no. 5, pp. 336-344, 1975.

[17] T. Bag and S. K. Samanta, "Finite dimensional fuzzy normed linear spaces," Journal of Fuzzy Mathematics, vol. 11, no. 3, pp. 687-705, 2003.

[18] H. Drygas, "Quasi-inner products and their applications," in Advances in Multivariate Statistical Analysis, A. K. Gupta, Ed., pp. 13-30, Reidel, Dordrecht, The Netherlands, 1987.

[19] V. A. Faiziev and P. K. Sahoo, "On the stability of Drygas functional equation on groups," Banach Journal of Mathematical Analysis, vol. 1, no. 1, pp. 43-55, 2007.

[20] A. Najati and M. B. Moghimi, "Stability of a functional equation deriving from quadratic and additive functions in quasi-Banach spaces," Journal of Mathematical Analysis and Applications, vol. 337, no. 1, pp. 399-415, 2008. 
[21] C. I. Kim, G. Han, and S.-A. Shim, "Hyers-Ulam stability for a class of quadratic functional equations via a typical form," Abstract and Applied Analysis, vol. 2013, Article ID 283173, 8 pages, 2013.

[22] A. K. Mirmostafaee, M. Mirzavaziri, and M. S. Moslehian, "Fuzzy stability of the Jensen functional equation," Fuzzy Sets and Systems, vol. 159, no. 6, pp. 730-738, 2008. 


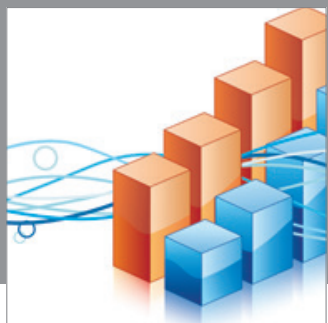

Advances in

Operations Research

mansans

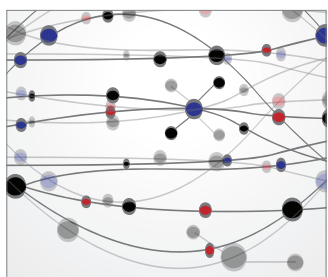

The Scientific World Journal
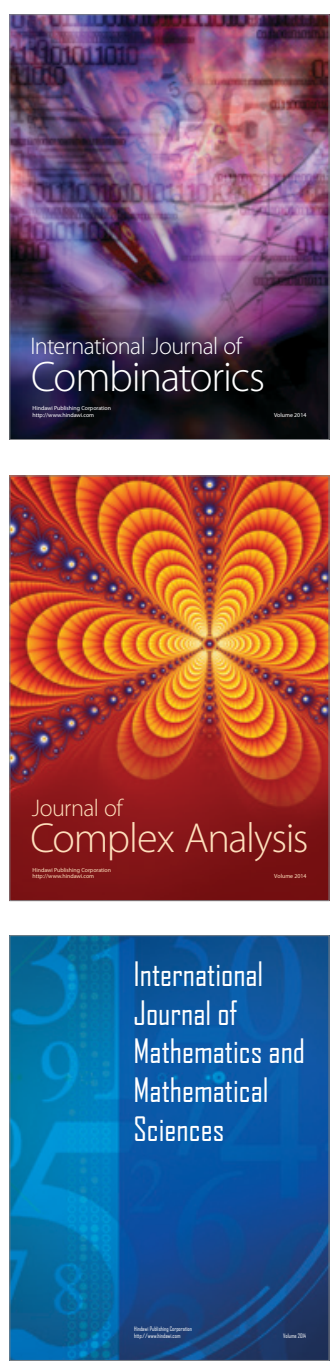
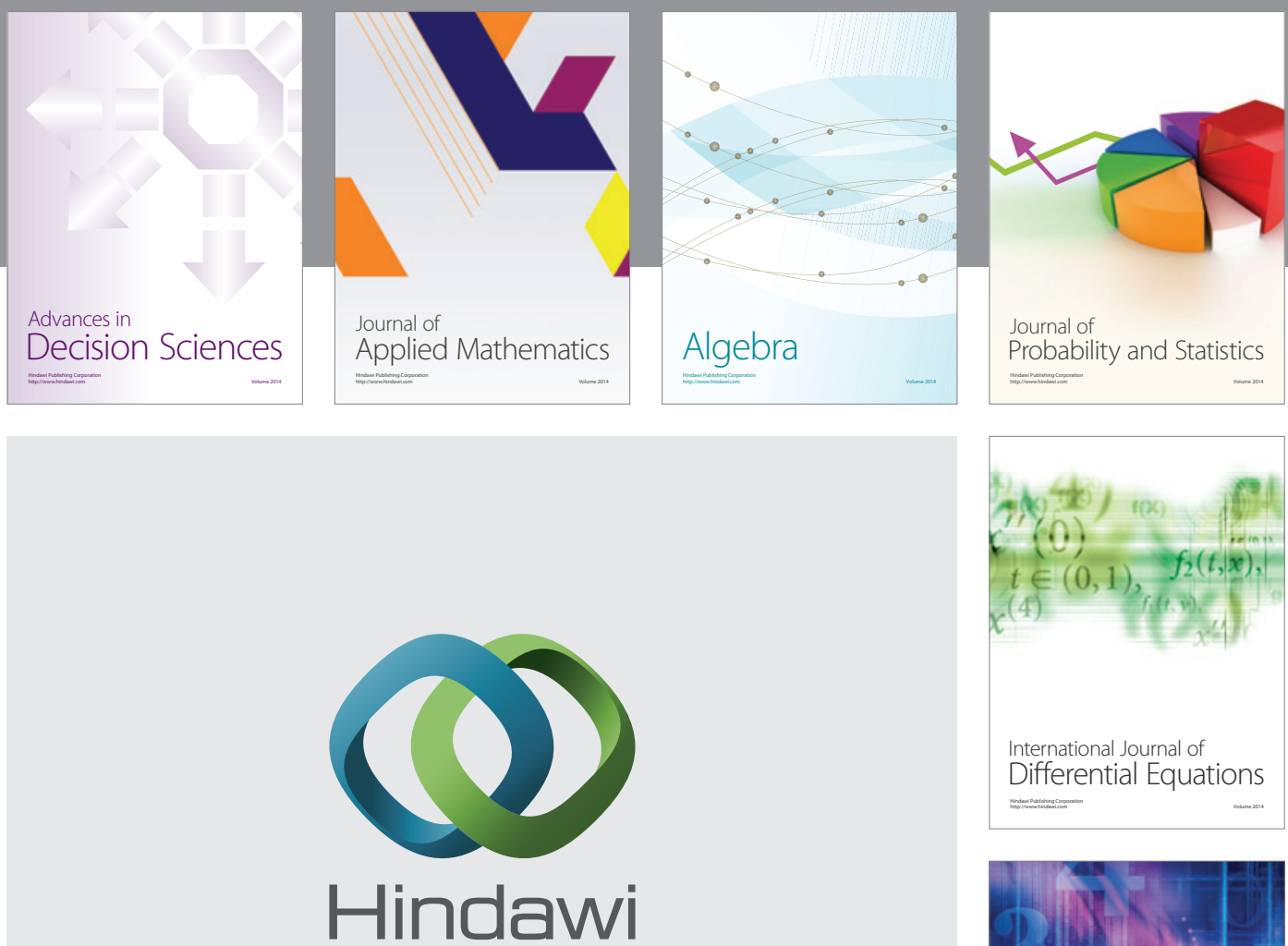

Submit your manuscripts at http://www.hindawi.com
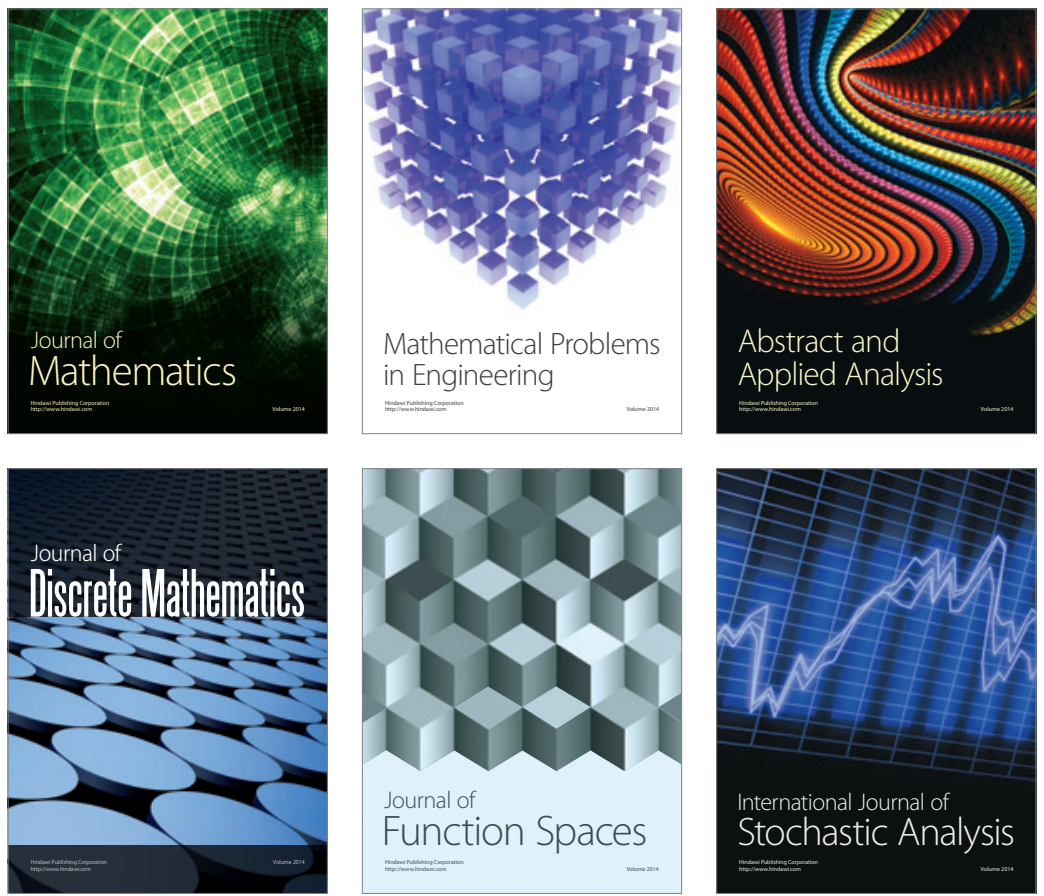

Journal of

Function Spaces

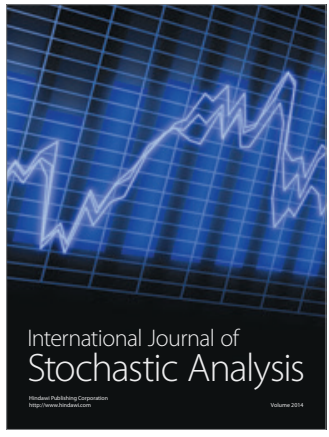

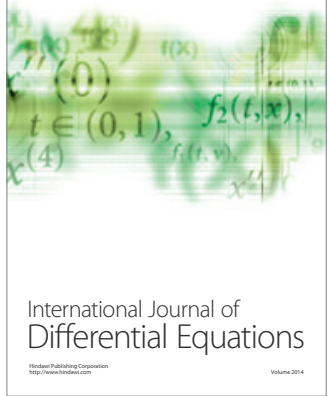
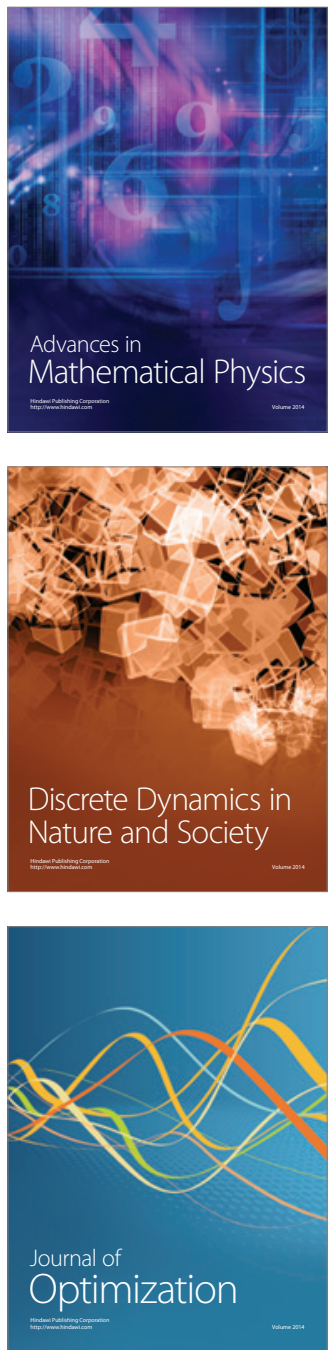